\title{
Treatment Options for Oral Submucous Fibrosis
}

\author{
Keerththana Balabaskaran ${ }^{1}$,Dr. Arathy Srinivasan. L ${ }^{2}$ \\ ${ }^{\prime}$ (Saveetha Dental college, India) \\ ${ }^{2}$ (Department of oral medicine, Saveetha Dental college, India)
}

\begin{abstract}
Oral submucous fibrosis is a chronic disease of oral cavity which is common in Indian subcontinent and caused by chewing areca nut and other irritants in various forms. It is a potentially malignant condition and is of major concern to oral physicians. A wide range of treatment has been attempted till date, but none of them are proved to be a complete cure for this disease. The aim of this review is to present an overview of various treatment modalities of oral submucous fibrosis and their rationale.
\end{abstract}

Keywords: Areca nut, Betal quid, Habits, oral sub mucous fibrosis, steroid therapy.

\section{Introduction:}

Oral submucous fibrosis is defined as the chronic, insidious disease affecting the oral cavity and sometimes pharynx, although occasionally preceded and/or associated with vesicle formation and is always associated with juxtaepithelial inflammatory reaction followed by fibro elastic changes in the lamina propria with epithelial atrophy leading to stiffness of oral cavity leading to trismus and inability to eat ${ }^{[1]}$.

Oral submucous fibrosis is a chronic debilitating and a well recognised potentially malignant condition associated with areca nut chewing, an ingredient of betel quid and is prevalent in South Asian population. Pathogenesis is not yet established but is believed to be due to multifactorial causes; hence the treatment of oral submucous fibrosis postulates a major challenge for oral physicians. Current article discusses the various treatment modalities available.

\section{Treatment Options:}

The main symptoms of oral submucous fibrosis include trismus, burning sensation, and difficulty to eat. Hence the treatment modalities have been tried to relieve these symptoms. It has been tried by both surgical and non surgical approach.

\section{Cessation Of Habit:}

The cessation of habit of eating betal quid, areca nut and other local irritants, spicy and hot food, alcohol and smoking through education and motivation. Affected patients should be warned about the possible malignant potential.

\section{Supplementary Care:}

Iron, vitamins and minerals rich diet should be advised to patients with oral submucous fibrosis. Various studies have implemented deficiency of iron both as a cause and effect in pathogenesis and etiology of OSMF.Hence routine haemoglobin levels followed by iron supplements should be give ${ }^{[2][3]}$.

According to Martin and Koop ${ }^{[4]}$ considered vitamin B deficiency to be important in the etiology of degenerative changes in oral mucosa before malignant transformation. Sirsat and khanolkar ${ }^{[5]}$ reported that the reaction caused by capsaicin in arousing a limited connective response was enhanced by vitamin B deficiency. Thus vitamin B complex administration may relieve glossitis, inflammation of tongue and cheilosis in OSMF patients.

Several studies have confirmed the cancer preventive nature of antioxidants. The oral intake of retinoid has significant toxic effect in normal tissue. A less toxic group of micronutrients are caroteniods which include lycopene, its mode of action may involve stimulation of immune system or direct action in tumour cells.Lycopene has been shown to inhibit hepatic fibro genes is LEC rats by Kitade et al, and it may also exert a similar inhibition on the abnormal fibroblasts in OSMF. The findings of this study are in concurrence with those of another study which was performed by Haque et al. Lycopene also up regulates the lymphocytes resistance to stress and suppress the inflammatory response $\mathrm{e}^{[6][7] .}$

\section{Steroid Therapy:}

Steroids inhibit the proliferation of fibroblasts and this cause reduction in the number of collagen fibres. They also act to release cellular proteases in the connective tissue extracellular compartment which in 
turn activate the collagens and zymogen that ingest the insoluble collagen stimulating the rate if collagen breakdown. They also act by inhibiting the inflammatory response ${ }^{[2]}$.

Steroid ointment is applied topically in the cases with ulcer and painful oral mucosa. It is a common practice in India to treat OSMF patient using intra lesional dexamethasone injection. In OSMF the oral mucosa is already atrophied and inflamed. By using intra lesional method, there be following disadvantages:

1) More discomfort to patient

2) Needle trauma may heal by fibrosis.

The various problems associated with intralesional injection could be solved by atraumatic method of drug delivery system like mucosal patch ${ }^{[8] .}$

\section{Hyaluronidase [9]:}

The combination of steroids and hyaluronidase shows better long term results than either agent used alone (Kaker 1985). It produces burning sensation and strismus.it acts by breaking down hyaluronic acid, lowers the viscosity of intracellular substances and decreases collagen formation.

\section{Pentoxifylline:}

Rajendran et al had tried earlier Pentoxifylline as treatment in patients with OSMF and reported good results (rajendran 2006). Pentoxifylline is a tri substituted methyl methylxanithine derivative. It is termed as rheological modifier; it improves micro circulation and decreased platelet aggregation as well as granulocyte adhesion $^{[11]}$. Haddad et al treated 34 radiation induced superficial fibrotic lesions of skin with Pentoxifylline and vitamin $\mathrm{E}$ for 3 months and reported a significant improvement in radiation induced fibrosis (haddad et al $2005)^{[10]}$. Rawlins et al reported that Pentoxifylline has a direct effect on inhibiting burn scar fibroblasts (Rawlins et al) ${ }^{[12]}$. Extrapolating this finding, the drug has been also used to alleviate the symptoms in patients with OSMF. There are both central nervous system and gastrointestinal system side effect which are dose related and therefore minimized by dose reduction.

\section{Placental Extracts: ${ }^{[14][2]}$}

Placentrix is an aqueous extract of human placenta that contains nucleotides, enzymes, vitamins, amino acids and steroids, it acts by: biogenic stimulation. Its use is based on the method by: tissue therapy introduces by filatov in 1933, later in 1953. His theory states that animal and vegetable tissue, when severed from parent body and exposed to conditions unfavourable but not mortal to their existence undergo biological re adjustments leading to development of substance in state of survival to ensure their vitality such tissue are the extract, implanted or injected in to the body after resistance to pathogenic factors stimulate the metabolic or regenerative processes, thereby favouring recovery. It has no contra indications and results are obtained to be lasting.

VIII. Chymotrypsin: [2]

Chymotrypsin, an endopeptidase, hydrolyses ester and Peptide bonds, thus acting as a proteolytic and anti-inflammatory agent.

\section{Interferon-Gamma ${ }^{[15]:}$}

This plays a role in treatment of patients with OSMF because of its immino regulatory effect. INTERFERON-GAMMA is also known as anti fibrotic cytokine, patients treated with an intralesional injection of interferon gamma experienced improvement of symptoms.

\section{Immune Milk ${ }^{[16][2]: ~}$}

Immune milk contains an anti inflammatory component that may suppress the inflammatory reaction and modulate cytokine production. Symptomatic relief in patients maybe partially attributed to mice nutrients contained in the immune milk powder.

\section{Turmeric ${ }^{[17][2]:}$}

Administration of turmeric powder offers protection benzoppyrene induced increase in micro nuclei in circulating lymphocytes and its excellent scavenger of free radical invitro. Turmeric oil and turmeric oleoresin both act synergistically in vivo to offer protection against DNA damage.

\section{Physiotherapy ${ }^{[2]}$ :}

Muscle stretching exercises for the mouth may be helpful to prevent further limitations of mouth opening forceful mouth opening has been tried with mouth gag and acyclic surgical screw. 


\section{Diathermy ${ }^{[2]}$ :}

Microwave diathermy seem superior to short wave, because selective heating of juxtaepitheliel connective tissue is possible it acts by physio fibrinolysis of bands.

\section{Ultrasound ${ }^{[18]}$ :}

Ultra sound selectivity raise the temperature in some well accumulated areas. Ultrasound proves to be an efficient deep heating modality.

\section{XV. $\quad$ Surgical Treatment ${ }^{[2]}$ :}

It is indicated in patients with severe trismus surgical modulates that have been used include simple excursion if fibrosis bands, with exacerbation of the condition. Split-thickness skin grafting following bacterial temporalis myotomy in coronidectromy.

\section{Lasers:}

$\mathrm{CO}_{2}$ lasers are predefined rather than a scalpel or technique involving multiple tiny incisions for surgical relief of limited oral aperture because the laser beam spontaneously seal all the blood vessels, allowing the surgeon perfect visibility and accuracy in excising fibrous tissue[19].

\section{Cryosurgery:}

It is the method of local destruction of tissue by freezing it insitu. Extreme cold is produced by liquid nitrogen or argon gas to destroy abnormal tissue.

\section{Conclusion:}

OSMF is one of the most poorly understood and unsatisfactorily treated oral diseases. All available treatments give patients only symptomatic relief which is short lived. Patients suffering from this incurable, chronic fibroblastic scanning disease need to be informed. It is essential at the onset of treatment to avoid rising exceptations.It still requires extensive research for complete treatment of OSMF.

\section{References:}

[1]. Pinborg J.J and Sirsat S.M Oral submucous fibrosis , oral surg oral med oral path 2001,22(6):764-79

[2]. Lavina, T.Anjana B and Vaishali K. Haemoglobin levels in patients with oral submucous fibrosis: JIAOMR 2007.19:02, 329-333.

[3]. Taneja L, Nagpal A, Vohra P, Arya V. Oral submucous fibrosis: an oral physician approach. Journal of innovative dentistry vol 1 issue 3 sept-dec 2011

[4]. Martin H.Koop EC. Precancerous mouth lesions of avitaminosis B; their etiology, response to therapy and relationship to oral cancer. Am.J surg 1942; 57:195.

[5]. Sirsat SM, Khanolkar VR. Submucous fibrosis of palate in diet -preconditioned Wistar rats. Induction by local painting of capsaicinan optical and electron microscopy study; Arch pathol 1960,70,171-9.

[6]. Kumar A, Bagewadi A, Keluskar V, Singh M. Efficacy of lycopene. Oral surg oral med oral pathol oral radiol 2007; 103:207-13.

[7]. Bhagavan Bangie komacy gowde, yathish TR, Srihasan san kappa P, Kumar Naik H, Purushothen somayaji, Anand D. Response of oral submucous fibrosis to lycopene- a caroteniods anti oxidant -a clinicopthological study. Journal of clinical and diag research 2011, august vol 5(3)

[8]. Efficacy of dexamethasone mucosal patch for osmf a pilot study. Prof KN sumanth, Prof Dr.Ravikiran ongole.Int poster J dent oral med 2010, vol 12 no2 poster 484.

[9]. Kakar PK, Puri PK and Venkatachalam VP. Oral submucous fibrosis. Treatment with hyalase J laryngol otol 1985, 95; 55-59.

[10]. Haddad, Kalaghchi B, Amouzegar- Hashemi F (2005). Pentoxifylline and vitamin E for superficial radiation induced fibrosis: a phase 2 clinical trial Radiother oncol, 77, 324-326.

[11]. Rajendran R, Rani V, Shaikh S (2006). Pentoxifylline therapy: a new adjuant in treatment of oral submucous fibrosis. Indian journal of dent research 17, 190-198.

[12]. Rawlins JM, Lam WL, Haroo RO et al (2006) Pentoxifylline inhibits mature burn scar fibroblastin culture Burns 32, $42-45$.

[13]. Pentoxifylline therapy in management of oral submucous fibrosis. Ravi mehrotra, HP singh, Sc gupta, M Singh, S Jain. Asian pacific journal of cancer prevention vol 12, 2011 Pg 971-74.

[14]. Chitko GA, oral submucous fibrosis: A clinical, histopath and therapeutical study MDS Thesis university of Bombay 1968

[15]. Haque MF, Meghji S, Nazir R and Harris M. Interferon gamma may reverse oral submucous fibrosis. J. Oral pathol med Jan 2001, 30:12-21.

[16]. Tai Y.S, Liu B.Y, Wang J.T, Sun A, Kwan H.W and chiang C.P. oral administration of milk from cows immunised with human intestinal bacteria leads to significant improvements of symptoms and signs in patients with Oral sub mucous fibrosis. J.oral pathol med 2001, 30:618-25.

[17]. Hastak K, lubri N, Jakhi SP et al. Effect of turmeric oil and turmeric oleoresin on cytogenic damage in patients suffering from oral submucous fibrosis Cancer letters 116; 265-9,1997

[18]. Bierman W, ultra sound in treatment of scars. Arch phy med rehabil 1954, 35, 209-214.

[19]. Frame J.W carbon dioxide laser surgery for benign oral lesions Br. Dent J. 1985;158;125-8 